\title{
Spatial-temporal heterogeneity of out-of-hospital cardiac arrest incidence in Swiss Canton Ticino
}

\section{Caputo ML1,2, Peluso $\mathrm{S}^{3}$, Reinhold $\mathrm{J}^{3}$, Burkart $\mathrm{R}^{4}$, Benvenuti $\mathrm{C}^{4}$, Cianella $\mathbf{R}^{5}$, Moccetti $\mathrm{T}^{1}$, Mira $\mathrm{A}^{3,6}$, Auricchio $\mathrm{A}^{1}$}

(1) Cardiocentro Ticino, Lugano, Switzerland; (2) Department of Molecular Medicine, University of Pavia, Coronary Care Unit and Cardiovascular Clinical Research Center, Fondazione IRCCS Policlinico San Matteo, Pavia, Italy ; (3) Data Science Center, Institute of Computational Science, Università della Svizzera italiana, Switzerland; (4) Fondazione Ticino Cuore, Breganzona, Switzerland; (5) Federazione Cantonale Ticinese Servizi Autoambulanze, Lugano, Switzerland; (6) Department of Science and High Technology, Università dell'Insubria, Italy

\section{Introduction}

Incidence and outcome of out-of-hospital cardiac arrest (OHCA) differs significantly across geographic regions. Past studies have indicated the existence of OHCA clusters at national or state level; much less is known for OHCA clustering at county or municipality level. Furthermore it is unknown whether OHCA clusters may change over years. Understanding these issues may have significant implications for OHCA management.

\section{Purpose}

We aimed to determine whether OHCA incidence was homogeneously distributed at municipality level in Swiss Canton Ticino, a large territory including rural and urban areas; to assess areas of increased risk of OHCAs over time, according to demographic characteristics of the resident population and to predict OHCA events at municipality level.

\section{Methods}

All data regarding OHCAs which occurred in Canton Ticino (Switzerland) from January 1, 2005, through to December 31,2017 were entered into a prospectively designed registry and geolocated. Demographic characteristics (sex composition and age distribution) at the level of single municipality were collected from the Federal Office of Statistics. Counting and incidences were calculated at level of each municipality, by taking into account gender and age distribution.

The Integrated Nested Laplace Approximation (INLA) was used for estimate OHCA incidences with related uncertainty in a training sample (OHCAs occurred between 2005 and 2015), and to predict OHCA incidences in a validation cohort (OHCAs occurred in 2016-2017).

\section{Results}

A total of 2129 OHCAs of presumed cardiac origin occurred in the 117 municipalities of Canton Ticino. Of those, 564 (27\%) had a VT or VF as presenting rhythm. There was a negligible annual fluctuation in the absolute number of OHCA without significant change over time of the overall OHCA incidence. OHCAs occurred prevalently at home (70\%), in men (68\%) of a median age of 71 years. We observed a concentration of cardiac events in the Southern part of Ticino, with a spatial distribution that followed the geographical configuration of valleys. As expected, the absolute numbers of OHCAs were higher in more populated areas, but some less populated municipalities showed relevant OHCAs incidence, that could be explained with a higher prevalence of male people with an age $\geq 80$ years old.

\section{Prevalence and incidence of OHCAs in Swiss Canton Ticino (2005-2015). Red to light yellow colour grading indicates municipalities with high to low prevalence or incidence, respectively}
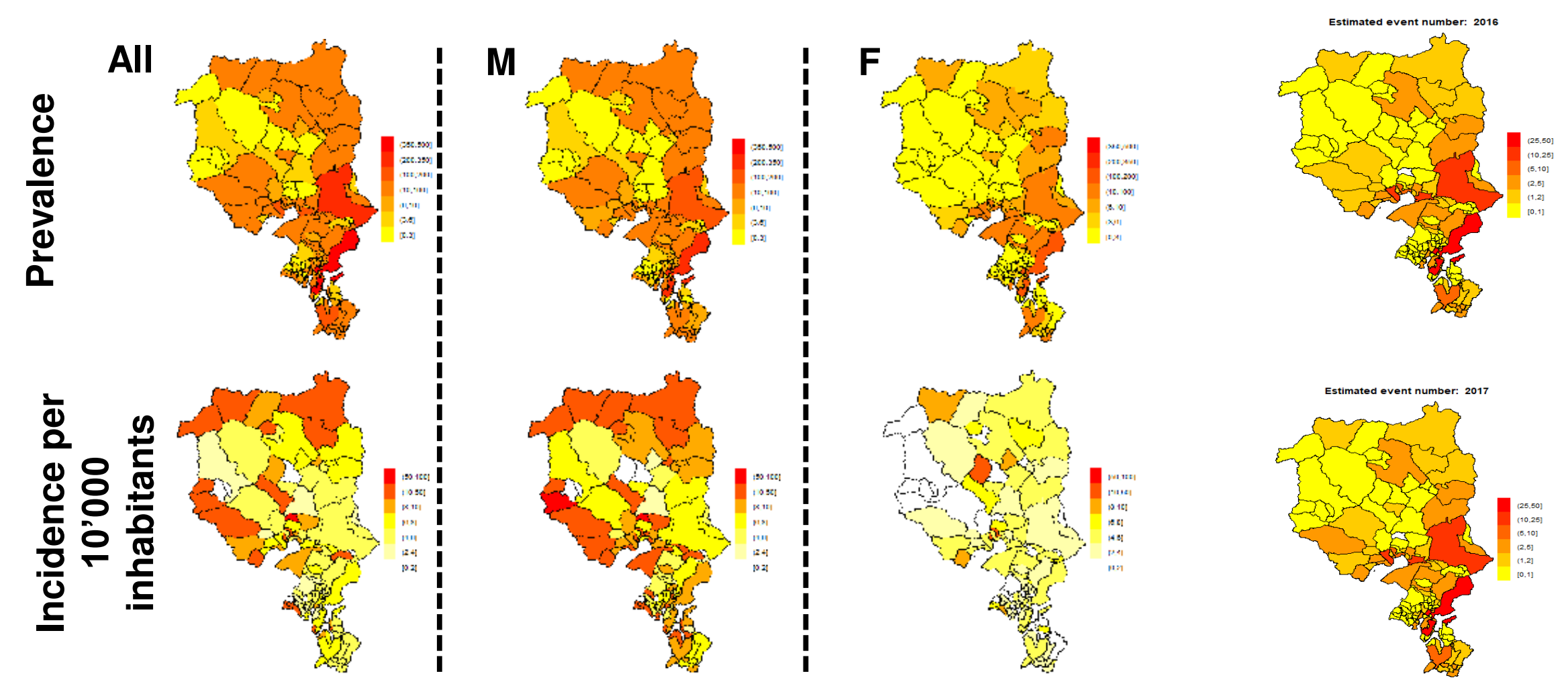
INLA model performance in prediction of OHCAs distribution in 2016 and 2017 years. Left maps: real distribution of OHCAs; central figure maps: predicted distribution; right panel histograms: Prediction errors bars

When tested in the validation cohort (years 2016-2017), the predictive model was able to capture roughly $90 \%$ of OHCAs variability. The model was able to predict OHCAs exactly (with null prediction error) in 51\% of all municipalities, and with a minor error of 1 to 2 events in the other municipalities.

\section{Conclusions}

Significant differences in OHCA prevalence and incidence among municipalities were observed. A predictive model taking into account territorial gender and age distribution is able to predict up to $90 \%$ of events variability. This approach provides a great opportunity for preventive measures and reduction in access time to OHCA. 